\title{
A COVID-19 Spread Model of the Discrete Grid to Assess the Potential of Non-pharmaceutical Interventions
}

Wen Cao ( $\sim$ jwzx_edifier@163.com )

Zhengzhou University https://orcid.org/0000-0003-2182-7819

Haoran Dai

Zhengzhou University

Xiaochong Tong

Zhengzhou University

Jingwen Zhu

Zhengzhou University

Yuzhen Tian

Zhengzhou University

Feilin Peng

Zhengzhou University

\section{Research Article}

Keywords: COVID-19, Epidemiological model, Non-pharmaceutical interventions, Spatiotemporal spread model of COVID-19

Posted Date: January 18th, 2021

DOI: https://doi.org/10.21203/rs.3.rs-143792/v1

License: (c) (1) This work is licensed under a Creative Commons Attribution 4.0 International License. Read Full License 


\section{A COVID-19 spread model of the discrete grid to assess the potential of}

2 non-pharmaceutical interventions

3 Wen Cao $^{1{ }^{* *}}$, Haoran Dai ${ }^{1 \dagger}$, Xiaochong Tong ${ }^{2}$, Jingwen Zhu' ${ }^{1}$, Yuzhen Tian ${ }^{1}$, Feilin Peng ${ }^{1}$

$4 \quad *$ Corresponding email: jwzx_edifier@163.com (Wen Cao)

5 Abstract

6 Background: The outbreak of COVID-19 posed a serious threat to human health, economic development, and social stability worldwide, and many countries had taken different interventions to control the deterioration of the epidemic. Although many studies have evaluated the effectiveness of these interventions, there were few reasonable explanations for the practical geographic significance of the model parameters. Our aim was to evaluate the potential of different interventions to mitigate the spread of the epidemic, including discussion about the different time and intensity of implementation, and map parameters of model to the practical application meanings of the special interventions.

Methods: In this study, a COVID-19 spread model based on the discrete grid was proposed from perspective of geography. A multi-level grid was introduced to describe the quarantine status and intensity in different spaces, which also combined with the model of medical reception-cured and self-protection, and the spatiotemporal evolution process of early COVID-19 spread was simulated based on the spatial correlation, finally, the effect of interventions was quantitatively analyzed by the dynamic transmission model of COVID-19.

Results: Quarantine measure were the most effective interventions, which could effectively reduce the peak value of infection, advance the arrival time of the peak, and shorten the duration of the epidemic, but it only played a role under sufficient intensity; the medical reception-cured and self-protection measure could effectively fatten the infection curve and slowed the spread of the epidemic in the early stage, which could provide more buffer time for the relevant government departments, but the practical effect was not obvious because of the limitation of actually invested resources. The role of the medical reception-cured measure was more reflected in the reduction of the number of deaths, and the effect of the self-protection measure could be reduced in strict quarantine measure.

Conclusions: Results of the study indicated that the quarantine, medical reception-cured and self-protection measures were effective, and mitigating the spread of COVID-19 by achieving strong interventions was necessary. Strict quarantine should be implementing as soon as possible in countries with serious development of COVID-19.

Keywords: COVID-19; Epidemiological model; Non-pharmaceutical interventions; Spatiotemporal spread model of COVID-19;

\section{Background}

In late December 2019, a novel coronavirus pneumonia epidemic caused by the SARS-CoV-2 virus (known as COVID-19) spread worldwide. Due to its high infection rate, long incubation period, and 
asymptomatic infection, the virus quickly spread widely among the population, which had brought an unprecedented blow to human life, economic development, and social stability ${ }^{[1-4]}$. In the absence of effective vaccines, countries usually need to implement effective intervention measures as soon as possible to alleviate the spread of COVID-19, such as strict quarantine measure, wearing face-mask, school closure, and large-scale tests ${ }^{[5]}$. However, the unknown and sudden nature of the virus makes it difficult for people to make a scientific and reasonable emergency plan policy as early as possible. Meanwhile, the large-scale personnel deployment and material distribution as well as the implementation of the policy plan also need a long time to prepare. Therefore, the implementation scope, implementation scale, intervention time, and potential effect of the prevention and control measures are key problems to be solved urgently in all countries under the condition of different degrees of COVID-19 spread, which will provide important support and guarantee for scientific and effective prevention and control of infectious diseases.

The early researches about the COVID-19 mainly focused on epidemiology, which could be roughly divided into three aspects: the estimation of the epidemiology parameters, the forecast of the epidemic situation, and the analysis of the effectiveness of intervention measures. In the early stage of the outbreak of COVID-19, most scholars estimated the basic reproduction number, incubation period, and other epidemic parameters by using the early reported case data and overseas export data, which was helpful to understand the dynamics spread mechanism of COVID-19 while preliminarily assessing the epidemic risk level ${ }^{[6-10]}$. Subsequently, more scholars began to predict the epidemic situation, and their main idea was to fit the reported case data by improving compartmental models. For example, the SEIQR、SIR-X and SIQR models considering the impact of isolation measures were proposed respectively ${ }^{[11-13]}$; a zonal SEIRD model combining the spatial diffusion and heterogeneity of infectious diseases was proposed $\left.{ }^{[14}\right]$; the SEIRS model with exponential structure was proposed ${ }^{[15]}$; some scholars have proposed new infectious disease models by adding asymptomatic infectors and environmental infection to SEIR model ${ }^{[16-20]}$; some stochastic based regression models were used to forecast the phenomena in as many as ten most affected states of Brazil ${ }^{[21]}$; a hybrid ARIMA-WBF model was considered to forecast different COVID-19 infected countries worldwide ${ }^{[22]}$; a three-stage e-ISHR introducing the time delay mechanism was established ${ }^{[23]}$; a discrete multi-stage dynamics system with time delay based on development process of China's epidemic was established ${ }^{[24]}$; the evolution of epidemic spread was simulated by introducing the statistical characteristics of complex network distribution into epidemiological model ${ }^{[25-26]}$. Most of these models 
focus on the time-series analysis of COVID-19 spread. The model parameters have a clear epidemiological significance, but lacking a spatiotemporal description of practical significance. With the good effect of epidemic prevention and control in some countries, many scholars began to evaluate the effect of different epidemic prevention and control measures on the alleviation of epidemic situation, such as quarantine ${ }^{[6,13,18,27-29]}$, wearing face-mask ${ }^{[16,30]}$, social distance ${ }^{[16,18,30-31]}$, travel restriction ${ }^{[32-33]}$, tracking and isolation of cases ${ }^{[16,34]}$, school closure ${ }^{[18]}$, protection of the elderly over 70 years old ${ }^{[18]}$, hospital isolation ${ }^{[23,35]}$ and external factor(ventilation and hand washing) ${ }^{[36-39]}$; some scholars evaluated the effect of interventions by making statistical analysis on the trend of epidemic data in several countries ${ }^{[40-42]}$. We found that most scholars mainly aimed to estimate the effectiveness of intervention measures by adjusting the model parameters to simulate the epidemic spread trend under different intervention measures based on the prediction analysis model. However, there was no reasonable explanation for the mapping between the model parameter values and the practical application meanings of special interventions, and few studies consider that the spatiotemporal evolution of COVID-19 may have different impacts on epidemic interventions. Besides, most of the models were mainly based on the compartmental model, taking the epidemic area as a whole, which lacked the expression of spatial information so that it is difficult to obtain the information about the spatial spread of COVID-19. Therefore, how to use the quantitative method to consider spatial information and the impact of interventions in the traditional classical compartmental model based on time-series process is the difficulty and focus of scientific research.

Geography is a discipline that studies the spatial distribution law, spatiotemporal evolution process, and regional characteristics of the earth's surface geographical elements or geographical complexes, which has been widely used in all walks of life. The spread and prevalence of COVID-19 is the result of a serious of complex natural and social factors, and one of its most important characteristics is spatiotemporal spread, which leads to understanding the phenomenon as geographical and potentially mappable ${ }^{[43-44]}$. At present, some scholars have researched in this field, such as the mobile phone data were used to forecast the epidemic situation in Wuhan from the perspective of spatial interaction ${ }^{[45]}$; the association between the American nursing home-level metrics and county-level, place-based variables with COVID-19 confirmed cases in nursing homes across the United States were established by using spatial modeling technology ${ }^{[46]}$; the ENM was utilized to assemble the epidemic data and nine socioeconomic variables to identify the potential risk zones in Beijing, Shenzhen, and Guangdong ${ }^{[47]}$. However, the detailed and complete 
observation data are usually difficult to obtain in a public health and safety emergency. Although these studies used multi-source data to analyze the epidemic situation, it still belonged to observation and reasoning under the small sample data because of the precision, resolution of data. Besides, most of the current epidemic data only include the location where the patients appeared symptoms, but the location where the patients were infected is rarely known, which indicates the current epidemic data also have a backtracking problem. Thus how to build observation strategies and scientific methods inline the reality is the key and difficult point of the current public health and safety emergencies in absence of detailed observation data ${ }^{[48]}$.

In this paper, we proposed a COVID-19 spread model in a discrete grid from the perspective of geography. The goals of the study are two-fold; firstly, to evaluate and quantify the impacts of interventions for mitigating the spread of COVID-19 by considering the spatial information, and secondly, to explain reasonably the practical significance of model parameters after mapped model parameters to the practical geographic area, which can provide better and more direct information to the public health and safety emergencies policy. The multi-level grid was used to divide the geographical space of the epidemic area, and the status and intensity of quarantine for the sub-region were described by boundary and size of the grid respectively. Then the medical reception-cured model was constructed by allocating medical capacity to the affected grid after considering the correlation between the spatial distribution of hospitals and the grid. Besides, the proportion parameters of the self-protection population (including wearing face-mask, washing hands frequently, ventilation, etc.) were introduced and mapped the corresponding grid regions to achieve modeling of self-protection measure. Considering the spread way of infectious diseases usually starts from the spatial adjacent to the spreading path and then gradually spreads to the surrounding region (the state of free spread without human control) ${ }^{[49]}$, the spatiotemporal spread evolution of infectious diseases was simulated through the radius of people's daily life based on the spatial correlation between grids when the detailed and precise population migration data (such as shared bicycle data, subway data, bus data) are often difficult to obtain, and then the COVID-19 dynamics model with asymptomatic infection was introduced for analyzing and quantifying epidemic spread situation in different interventions. Since the epidemic prevention and control measures abroad are relatively late and loose ${ }^{[19]}$, we used the early reported cases data of the UK, US, Spain, and Germany to discuss the free spread status of COVID-19 in Wuhan city under the absence of early epidemic data of Wuhan caused by sooner 
interventions. Then the potential of quarantine measure, medical reception-cured measure, and self-protection measure to alleviate epidemic spread under different scales and different intervention times were discussed. Finally, the rationality and correctness of the model were evaluated with the actual epidemic data, hospital distribution data, and medical attribute data in Wuhan. The novelties of this study are highlighted as follows:

I.The association between the model parameters and the geographical space are established from the perspective of geography, which makes model parameters can be mapped to the practical application meanings of special interventions and provide more direct and effective information for the formulation of prevention and control policies after evaluating and quantifying the potential of interventions;

II.According to the law that infectious diseases gradually spreads to the surrounding region starting from the spatial adjacent to the infectious source, the spatiotemporal spread and evolution model of COVID-19 is established in the lacking detailed observation data of crowd dynamics;

III.The potential effect of the medical reception-cured measure on epidemic prevention and control is evaluated by allocating the capacity of medical reception and cure to the corresponding infected regions from the perspective of hospital distribution.

\section{Methods}

\section{1 data}

On December 31, Wuhan reported a "viral pneumonia of unknown", and then Wuhan issued a ban on "City closure". The government successively issued a series of measures, such as restricting transportation, school closure, closing entertainment places, and prohibiting public gatherings. At the same time, many designated hospitals such as "huoshenshan" and "leishenshan" as well as fangcang shelter hospitals began to be put into use. Medical staff and medical resources from all over the country have been involved in the fight against COVID-19 in Wuhan. In order to discuss the effect of quarantine, medical reception-cured and self-protection measure to alleviate spread of infectious disease, this study collected the global epidemic data including the number of confirmed cases per day, cumulative number of cured people and deaths from dingxiangyuan (https://ncov.dxy.cn/ncovh5/view/pneumonia) and COVID-19 Data Repository by the CSSE at Johns Hopkins University ${ }^{[50]}$ (https://www.arcgis.com/apps/opsdashboard/index.html\#/bda7594740fd4 0299423467b48e9ecf6); the data of designated hospitals in Wuhan, including the spatial distribution of hospital, intervention times, number of open beds and cumulative number of patients from Wuhan 
Municipal Health Commission, People's Daily and Baidu baike; and the data of medical resources in Wuhan, including the number of local registered medical staff, the number of supporting medical staff in different regions and the number of face-mask (including N95 masks and medical-surgical masks) from the website of Hubei Provincial Bureau of Statistics, Hubei Provincial People's Government and People's Daily.

When Wuhan city was closed for three weeks (February 12, 2020), the nucleic acid detection reagent method was changed to clinical diagnosis (including CT method), which caused large fluctuations in the cumulative confirmed cases. This problem was analyzed through Richards nonlinear curve model ${ }^{[50]}$, and according to the incubation period of COVID-19 for 7 14 days, the calibrated daily number of new confirmed cases from January 29, 2020, to February 11, 2020, was shown by Table 1.

< Table 1 Calibration data of newly reported cases per day in Wuhan >

2.2 Quarantine model based on the discrete grid

Quarantine measure usually takes "province - city - district - street - community - village - home" as the basic unit to limit people's activity regions, which greatly reduce the contact between the infected and susceptible populations to achieve the effect of restraining the spread of infectious diseases. However, the uncertainty of the size and irregular shape of the traditional prevention and control unit will bring great difficulties to the modeling, and the more precise administrative division data usually is not easy to obtain. According to the spatial characteristic of physical quarantine measure, the discretization of ground space was realized by the multi-level global discrete grid system with different size and series grid units. An adaptive statistical unit could be formed by the hierarchical geographic grid to fill the traditional prevention and control unit. As shown in Fig.1, the effective grid regions were the effective areas within the administrative division. The boundary and size of the grid could be used to describe the status and intensity of quarantine in a region respectively, and the distribution of the grid could map the practical implementation scope of quarantine measure.

< Figure 1 Grid filling diagram of traditional prevention and control unit >

The specific design is as follows: Let the spread area of infectious diseases be $A$, and the research areas were divided into sub-regions using the discrete grid, in which the total number of sub-regions was denoted by $n$. Referring to the model of COVID-19 spread dynamics ${ }^{[16]}$, the total human population in the epidemic area, denoted by $N$, was split into susceptible humans $S(t)$, exposed humans $E(t)$, asymptomatic 
infectious humans $A(t)$, symptomatic infectious humans $I(t)$, detected infected humans via testing $C(t)$ and recovered humans $R(t)$. The boundary of the grid was represented with a dotted line before the implementation of the quarantine measure (Fig.2). At this time, there were few restrictions on population flow, and the spread of COVID-19 was a state of free spread. The numerical changes of each population during the spread of COVID-19 could be quantified by the epidemic dynamic model of the whole region, as shown in the following equations 1 6. The related variable parameters of the model are described in Table 2.

$S_{t}=S_{1}-\sum_{j=1}^{t-1}\left(\frac{\beta_{0}\left(\alpha A_{j}+I_{j}\right)}{N-C_{j}} S_{j}\right)$

$E_{t}=E_{1}+\sum_{j=1}^{t-1}\left(\frac{\beta_{0}\left(\alpha A_{j}+I_{j}\right)}{N-C_{j}} S_{j}-\sigma E_{j}\right)$

$A_{t}=A_{1}+\sum_{j=1}^{t-1}\left(v \sigma E_{j}-\left(\theta+\gamma_{a}\right) A_{j}\right)$

$I_{t}=I_{1}+\sum_{j=1}^{t-1}\left((1-v) \sigma E_{j}-\left(\varphi+\gamma_{o}+d_{o}\right) I_{j}\right)$

$C_{t}=C_{1}+\sum_{j=1}^{t-1}\left(\theta A_{j}+\varphi I_{j}-\left(\gamma_{c}+d_{c}\right) C_{j}\right)$

$R_{t}=R_{1}+\sum_{j=1}^{t-1}\left(\gamma_{c} C_{j}+\gamma_{a} A_{j}+\gamma_{o} I_{j}\right)$

When the quarantine measure was implemented, all grid boundaries changed from the dotted line to solid lines, and the current time was denoted by $t_{0}$. The activities of the population would be limited in the sub-regions so that COVID-19 could only spread in the grid regions and couldn't affect the other grid regions (Fig.2). The number of different humans in the sub-grid was denoted by $S_{i}^{t} 、 E_{i}^{t} 、 A_{i}^{t} 、 I_{i}^{t} 、 C_{i}^{t}$ and $R_{i}^{t}$ at time $t$ respectively, and the numerical changes of each human were the sum of all sub-regions, as shown in the following equation $7 \sim 12$, where $N_{i}$ was the number of population in each grid, and its specific value was obtained from the statistical yearbook data using Kriging interpolation ${ }^{\text {[49] }}$.

$S_{t}=\sum_{i=1}^{n}\left(S_{i}^{t_{0}}-\sum_{j=1}^{t-1} \frac{\beta_{0}\left(\alpha A_{i}^{j}+I_{i}^{j}\right)}{N_{i}-C_{i}^{j}} S_{i}^{j}\right)$ 
$A_{t}=\sum_{i=1}^{n}\left(A_{i}^{t_{0}}+\sum_{j=1}^{t-1}\left(v \sigma E_{i}^{j}-\left(\theta+\gamma_{a}\right) A_{i}^{j}\right)\right)$

$I_{t}=\sum_{i=1}^{n}\left(I_{i}^{t_{0}}+\sum_{j=1}^{t-1}\left((1-v) \sigma E_{i}^{j}-\left(\varphi+\gamma_{o}+d_{o}\right) I_{i}^{j}\right)\right)$

$C_{t}=\sum_{i=1}^{n}\left(C_{i}^{t_{0}}+\sum_{j=1}^{t-1}\left(\theta A_{i}^{j}+\varphi I_{i}^{j}-\left(\gamma_{c}+d_{c}\right) C_{i}^{j}\right)\right)$

$$
R_{t}=\sum_{i=1}^{n}\left(R_{i}^{t_{0}}+\sum_{j=1}^{t-1} \gamma_{c} C_{j}+\gamma_{a} A_{j}+\gamma_{o} I_{j}\right)
$$

< Figure 2 Schematic diagram of the quarantine measure model based on a discrete grid >

< Table 2 Parameters description of COVID-19 spread dynamics model >

2.3 Spatiotemporal spread model of COVID-19 based on the discrete grid

The most important character of infectious diseases is that their spread needs certain ways of spread, such as air spread, droplet spread, close contact, and blood spread. Government departments usually take prevention and control measures to reduce the frequency of contact between infected humans and susceptible humans, such as strict quarantine, testing, and tracking of cases, wearing face-mask, and actively keeping distance with fever patients. Thus we need to mater more information about the spread of infectious diseases to evaluate the effect of prevention and control measures, that is, the distribution of the number of infectious humans in each grid at different times.

The differences of regions and virus type make the spatiotemporal spread mode, spread path, and spread capacity of infectious diseases have certain differences, but, in general, the spread of infectious diseases usually gradually spreads to the surrounding regions starting from the place adjacent to the spread path space ${ }^{[49]}$. The number of newly infected people in a sub-region at a certain time is not only affected by the number of infectious people in the previous time but also affected by the number of infectious people in the surrounding regions in the previous time when the population flow in the epidemic area is not completely limited, in other words, the infected grid includes the status of self-infection and mutual infection (Fig.3). Thus the epidemic area was intersected with the buffer of infected grids constructed by the people's average living radius to get the daily new infected grid regions, which denoted by $r$, and the 
incompletely infected grid was regarded as infected for the convenience of calculation.

In the process of the spread of infectious diseases, the number of newly infected cases per day always has a time-series change process that increases first and then decreases, and the infected people are composed of many discrete points in space. According to Tobler's first law of geography, the spread of infectious diseases restricted by geographical spatial factors has a strong spatial correlation characteristic ${ }^{[44]}$. The distance was used to describe the spatial weight concept of the infected grids, which could allocate the number of newly infected people at the next moment to the corresponding infected grid, as shown in Fig.3 and equations 13 14.

$W_{i}^{t}=\left(h_{i j}^{t}\right)^{-p} / \sum_{i=1}^{n^{t}}\left(h_{i j}^{t}\right)^{-p}$

$$
h_{i j}^{t}=\sqrt{\left(x_{i}^{t}-x_{j}^{t-1}\right)^{2}+\left(y_{i}^{t}-y_{j}^{t-1}\right)^{2}}
$$

where $W_{i}^{t}$ is the weight for the number of newly infected people assigned by the infected grid, $h_{i j}^{t}$ is the distance from the infected grid to the affected grid, $\left(x_{i}^{t-1}, y_{i}^{t-1}\right)$ is the coordinates of the center point of the infected grid, $\left(x_{j}^{t-1}, y_{j}^{t-1}\right)$ is the coordinates of the center point of the affected grid, and $n^{t}$ is the number of the affected grid, and $p$ is any positive real number, usually 2.

< Figure 3 Spatiotemporal spread model for infectious diseases based on a discrete grid > 2.4 The model of Self-protection measure During the outbreak of COVID-19, some common self-protection measures of the susceptible humans and infected humans can reduce the spread rate of COVID-19, such as wearing face-mask effectively, washing hands frequently, keeping ventilation in the house, and keeping distance with fever patients actively. Clinical and infectious disease studies have shown that proper self-protection measures could effectively reduce the risk of infection and the external spread of the virus by $70 \% \sim 80 \%{ }^{[52]}$. In this study, a parameter $\varepsilon$ was introduced to represent the population proportion of effective self-protection in the total population and could be mapped to each grid region. The infected probability of susceptible humans with the effective self-protection was reduced to $30 \%$ of the normal value, while the infected rate and external spread rate of other people remained unchanged (Fig.4A). 
2.5 The model of medical reception-cured measure

During the spread of COVID-19, the government opened designated hospitals and set up outpatient clinics to detect and receive suspected patients. People usually took the nearby hospital to diagnosis when they found themselves uncomfortable under the influence of people's living, work, activity range, and the surrounding environment. Therefore, the spatial distribution of hospitals and the number of beds has a great impact on the spread of infectious diseases, and there is a strong spatial correlation between the geographical space and hospital. Considering the open time lag of medical beds and government investment in medical beds is usually based on the number of currently infected people, the percentage parameter $\delta$ for the number of infected people was introduced to simulate the number of beds put into use in the hospital every day, and then the number of cases admitted to hospital every day will be allocated to the corresponding infected grid based to the spatial distance weight (Fig.4B), where $Q_{A}$ and $Q_{I}$ are the number of asymptomatic and symptomatic infected people admitted in the hospital every day respectively, and specific values are assigned to $\sum_{i=1}^{n} Q_{i}^{t}$ according on the corresponding detection ratio ( $\theta$ and $\varphi$ ). The number of daily medical reception-cured patients in each grid is shown in the following equations 15 17.

$\mathrm{h}_{i j}=\sqrt{\left(x_{i}-x_{j}\right)^{2}+\left(y_{i}-y_{j}\right)^{2}}$

$W_{i j}=\mathrm{h}_{i j}^{-p} / \sum_{j=1}^{n_{i}} \mathrm{~h}_{i j}{ }^{-p}$

$Q_{i}^{t}=\left\{\begin{array}{l}0, t \leq t_{j}^{0} \cup t \geq t_{j}^{1} \\ \sum_{j=1}^{H} w_{i j} \times \delta \times\left(A_{t}+I_{t}\right), t_{j}^{0} \leq t \leq t_{j}^{1}\end{array}\right.$

where $Q_{i}^{t}$ is the number of patients admitted by the hospital in the grid (asymptomatic and symptomatic), $h_{i j}$ is the distance between the grid and the hospital, $w_{i j}$ is the assigned weight of the number of hospital patients in the corresponding grid, $\left(x_{i}, y_{i}\right)$ is the coordinates of the central point of the infected grid, $\left(x_{j}, y_{j}\right)$ is the coordinates of hospital, $n_{i}$ is the number of the grid affected by the hospital, and $t_{j}^{0} t_{j}^{1}$ are the intervention time and closing time of the hospital respectively.

< Figure 4 Schematic diagram of medical reception-cured measure (A) and self-protection measure (B) > 


\section{Results}

272

3.1 Numerical simulation and analysis for the original spread state of COVID-19

In this section, the possible trend of the epidemic without any interventions in Wuhan was discussed to prepare the subsequent assessment for the impacts of intervention measures to mitigate the spread of the COVID-19 epidemic. The first thing to be clear is that no country will allow infectious diseases to spread freely, which means that the original spread state of any infectious diseases is usually not available. At present, many scholars used the early epidemic data of the study area or random sampling of the basic reproduction number $R_{0}$ to solve this issue ${ }^{[16,18]}$. Unfortunately, Wuhan is the first city in the world to openly face the COVID-19 epidemic. Due to the unknown characteristic of the virus and the rapid and intensive intervention measures, the number of early data samples in Wuhan are too few and cannot truly describe the early spread trend of the COVID-19. However, interventions in foreign regions are relatively late and loose ${ }^{[19]}$, which makes it is more likely that their early epidemic data is consistent with the real spread situation of COVID-19. But, the differences in the extent of COVID-19 spread, patterns of population movement, and levels of economic development in different regions make it difficult to state objectively and accurately which country's data are most appropriate for simulating the spread trend of COVID-19. Therefore, these studies selected the early epidemic data of the UK, the US, Spain, and Germany to discuss the original spread situation of COVID-19.

Our simulation is based on the genetic algorithm to estimate the parameters by regarding the daily number of confirmed patients as the adaptive index, and the other parameter values of the COVID-19 spread dynamic model are shown in Table 3. Clinically, the average incubation period of COVID-19 is 5.2 days, ranging from 3 to 14 days. We assumed that the data within 14 days after the strict measures are still early data, which was to avoid the phenomenon that the early sample data was too small to be estimated accurately. It is worth noting that our estimation started from the announcement of the first confirmed case in each country, but there were already unknown numbers of infected and exposed humans in the population at that time. Therefore, the estimation parameters included the estimation of the first day exposed cases $E_{1}$, asymptomatic cases $A_{1}$ and symptomatic cases $I_{1}$. We repeated the genetic algorithm 100 times to ensure the reliability of the results. The results are shown in Fig.5, and the corresponding estimated parameter values are shown in Table 4. Here, the time of early epidemic data in the UK, the US, Spain, and Germany is from January 31, 2020 to April 3, 2020, January 20, 2020 to March 28, 2020, February 1, 2020 to March 28, 
< Table 3 Values of parameters in the COVID-19 spread dynamics model epidemic model >

$<$ Figure 5 The number of newly reported cases per day of original state simulated by genetic algorithm (Small chart section: the red histogram represents the early epidemic data of each country, and the curve is the original spread state curve of COVID-19 in each country. Here, the starting time of X coordinate in the UK, the US, Spain, and Germany is January 31, 2020, January 20, 2020, February 1, 2020, and January 27 , 2020, respectively; big graph section: the solid-line curve is the original spread state curve of Wuhan. The red dotted-line curve shows the actual number of newly reported cases in Wuhan after calibration, and the purple dotted-line curve represents the actual number of newly reported cases in Wuhan. Here, the starting time of X coordinate in the Wuhan is December 28, $2020^{[9]}>$

<Table 4 Parameters of the COVID-19 original spread state curve estimated by genetic algorithm>

Fig.5 shows that: the results of the model to fit the early data of each country were better (small chart section in Fig.5). After bringing the fitting parameters to the COVID-19 dynamic model of Wuhan, the original spread state of COVID-19 compared to the real curve in Wuhan was mainly reflected in the improvement of peak value, the extension of peak arrival time, and the continuous-time of epidemic situation. However, although the early data was fit well (the small part in Fig.4), the future trend of each curve was quite different, mainly in the number of peaks. This difference was because the spread of the early epidemic was easily affected by the population distribution, population flow, and the efficiency of medical resource detection in different regions. Alberti et al. also pointed out that there was great uncertainly in using early data to predict the epidemic situation, and there is a big scientific verification problem in forecasting with small sample data ${ }^{[53]}$.

Table 4 shows that infection rate $\beta_{0}$, asymptomatic detection rate $\theta$, symptomatic detection rate $\varphi$, and basic reproduction number $R_{0}$ in the estimated parameters were roughly the same (the same countries), among the estimation of $R_{0}$ was similar to $\mathrm{Wu}^{[6]}$, Zhou ${ }^{[7]}$ and Wang ${ }^{[9]}$. However, the estimated values of $E_{1} 、 A_{1}$ and $I_{1}$ were quite different, mainly because it was difficult to confirm the actual number of infected people who had already exited but had not been tested or had no symptoms in the population when the first case was found. 
US, Spain, and Germany for the COVID-19 dynamic spread model of Wuhan to make up for the difficulty

of obtaining the original spread state of COVID-19 in Wuhan, and then try to estimate the effect of different interventions to mitigate the spread of the COVID-19, which eliminated the difference for potential assessment of prevention and control measures under different COVID-19 original spread.

3.2 Experimental analysis for the influence of different quarantine measure to mitigate the spread of COVID-19

This part mainly discussed the influence of quarantine measure with different intensities and intervention times to mitigate the spread of the COVID-19. The grid size represented the strength of the quarantine measure, that is, the corresponding quarantine measure became more stringent with grid size became finer. We took the intervention time when Wuhan city issued a ban on "city closure" as the baseline, and set the grid size to $1000 \mathrm{~m}, 500 \mathrm{~m}, 250 \mathrm{~m}, 100 \mathrm{~m}$, and $10 \mathrm{~m}$ respectively (Fig.6A). Then, we fixed the grid size of $10 \mathrm{~m}$ and adjusted the intervention time into 41 st day, 44th day, 47th day, 50th day, and 53rd day respectively (Fig.6B). The average daily life radius of people was $2000 \mathrm{~m}$, which was based on the travel characteristics of Wuhan residents ${ }^{[54]}$.

< Figure 6 The change curve of newly reported cases per day in Wuhan under different quarantine measure >

Fig.6A shows that: whatever the scale of the original spread curve of COVID-19, the curves would be close to the actual epidemic curve of Wuhan with the decrease of grid size, which was mainly reflected in the reduction of peak infection, the shortening of peak arrival time and epidemic duration. No matter what kind of original infection state, the original spread curves of COVID-19 was almost not changed at $500 \mathrm{~m}$ grid. This mainly because the spread of infectious diseases are easily affected by population flow and population distribution, but the $500 \mathrm{~m}$ grid does not limit the contact of the population, which shows that quarantine measure must reach a certain intensity to be effective, especially the home quarantine state (10 m grid). From the perspective of the severity of different original spread state, the peak infection, the time of peak arrival, and the duration of the epidemic situation were greatly reduced for the curve with a very serious spread degree. However, although the curve with a relatively light original spread state would decrease with the increase of grid size, it seems that the scale of reduction was much smaller. Thus we suggest that quarantine measure is a very effective epidemic prevention and control measure, especially for viruses with high infectious characteristics, and it also seems to be not necessary to use when the epidemic situation is relatively light because strict quarantine measure needs to invest unimaginable financial, 
material and human resources. This seems to have been demonstrated in South Korea and Japan ${ }^{[5]}$. From the perspective of the final results of the $10 \mathrm{~m}$ grid, even if the original spread trend of all curves were different, the final curves almost coincided. This is mainly because the role of quarantine measure is to protect the uninfected population by limiting the activities of the population. When the grid is very small, there are few people that infectious people can contact so that most of the uninfected people are protected, which makes the number of final infected people is almost the same.

Fig.6B shows that: the epidemic curve deviated from the actual curve in Wuhan with the delay of intervention time, which mainly reflected in the increase of peak value, the extension of peak arrival time, and epidemic duration. Compared with the actual epidemic curve in Wuhan, the number of new confirmed cases per day had more than doubled just one week later, which also emphasizes that quarantine measure need timely intervention to achieve better results.

3.3 Experiment analysis for the influence of different self-protection measure to mitigate the spread of COVID-19

This part mainly discussed the influence of self-protection measure with different proportions of effective self-protection population and quarantine intensities to mitigate the spread of the COVID-19. We took the intervention time when a group of pneumonia cases with unknown etiology were first published in Wuhan municipal health commission (January 11, 2020) as the baseline, and set the proportion of self-protection population to $0.1,0.2,0.3$, and 0.4 of the total human respectively (Fig.7A). Then, we adjusted the proportion of self-protection personnel to $0.1,0.2,0.3$, and 0.4 under the grid size of $500 \mathrm{~m}$, $250 \mathrm{~m}, 100 \mathrm{~m}$, and $10 \mathrm{~m}$, respectively (Fig.7B).

< Figure 7 The change curve of newly reported cases per day in Wuhan under different self-protection measure>

Fig.7A shows that: the original spread curve of COVID-19 gradually moves away from the actual curve of Wuhan with increasing the proportion of the self-protection population. However, it seemed that only the duration of the epidemic and the time to reach the peak would only increase, but the peak of infection was gradually declining. This is mainly because self-protection measure does not fundamentally cut off the spread of the virus, and only reduce the infected probability. The total population of Wuhan is about 14 million. Even if it is based on the ratio of 0.1 , the number of people who need to achieve effective self-protection every day in Wuhan will reach 1.4 million. In terms of the number of face-mask, this is an unimaginable expense. Therefore, we suggest that the role of self-protection measure mainly is to delay the 
arrival of epidemic peak and strive for more preparation time for the government.

Fig.7B shows that: under the grid of $500 \mathrm{~m}$ and $250 \mathrm{~m}$, the peak value of the COVID-19 spread curve decreased and the time to reach the peak value was delayed with the increase of the proportion of effective self-protection population. However, the COVID-19 spread curve hardly changed under the grid of $100 \mathrm{~m}$ and $10 \mathrm{~m}$. This is mainly because the strict quarantine measure makes the movable area of infectious patients very small, and self-protection measure is difficult to achieve an effect at this time, which also shows that some resources of self-protection measure, such as face-masks, medical clothes, hand sanitizer, and disinfectant, can be concentrated in the hands of high-risk groups who have to go out or need to contact with infected patients, such as medical staff, material distribution staff and relevant leaders under strict quarantine measure.

3.4 Experiment analysis for the influence of different medical reception-cured measure to mitigate the spread of COVID-19

This part mainly discussed the influence of medical reception-cured measure with different medical beds and intervention time to mitigate the spread of the COVID-19, and the number of medical beds was calculated according to the percentage of daily existed infected people (including symptomatic cases and asymptomatic cases). We took the intervention time when the first batch of designated hospitals in Wuhan (January 20, 2020), and set the proportion of medical beds to $0.1,0.2,0.3$, and 0.4 respectively (Fig.8A \& Fig.8B). Then, we fixed the ratio of bed investment was 0.1 and adjusted the intervention time into 34th day, 37th day, 40th day, 43rd day, and 46th day, respectively (Fig.8C).

< Figure 8 The change curve of newly reported cases per day in Wuhan under the different medical reception-cured measure (the length of the yellow vertical line and the green prism points indicates the 95\% confidence interval and the median values of the total number of invested beds corresponding to 10 curves in group B, respectively) >

Fig.8A shows that: the original spread curve of the COVID-19 decreased at the peak with the increase of beds (the proportion increased), which emphasized the effectiveness of the medical reception-cured measure. However, the time of peak arrival and the duration of the epidemic did not seem to change. This is mainly because there are a large number of undetected or ineffectively quarantined cases in the population when the medical reception-cured measure is put into use. Thus the medical bed will soon reach full load because of the limitation of the medical beds. Fig.8B shows the total number of medical beds required to be 
invested was about 580000 at the proportion of 0.1 , which is difficult to meet in reality.

Fig.8C shows that: the original spread curve shifted to the left with the intervention time moved forward, which was mainly reflected in the increase of peak arrival time and epidemic duration, but the peak did not seem to change. After maintaining a low level of epidemic spread for short time, the medical beds would reach the load, and the epidemic would break out again on a large scale. At this time, the medical system has collapsed and the scale of the outbreak again was similar to the original spread state, and the US seems to be facing the same problem. Therefore, we suggest that other effective measures must adopt to reduce the pressure on the medical system.

3.5 Model validation under actual interventions of COVID-19 in Wuhan

In this part, the actual quarantine intensity, the number of medical beds, and the proportion of effective wearing face-mask (self-protection measure) were used to verify the reliability of the model. In terms of quarantine measure, we used the $10 \mathrm{~m}$ grid to approximately represent the home quarantine measure implemented in Wuhan, and the intervention time was January 24, 2020, when Wuhan issued a ban on "lockdown". For the medical reception-cured measure, there were 68 designated hospitals in Wuhan, including 16 fangcang shelter hospitals, "huoshenshan" and "leishenshan" hospitals, with a total of 38782 beds. Thus our proportion of beds was approximately 0.06 of the daily existed infected people in the different original spread curves (including symptomatic and asymptomatic), and the intervention time was the time when the first batch of designated hospitals began to put into operation (January 20, 2020). Due to the number of humans complying with the self-protection measure was difficult to obtain, the proportion of self-protection measure in Wuhan was represented by the proportion of effectively wearing face-mask. We assumed each medical staff used two face-masks every day and every citizen consumed one face-mask every three days. Thus Wuhan needs to consume 2.1744 million face-masks per day referring to the total number of medical staff in Wuhan was about 108720 (including local medical staff and support medical staff) and the total population of Wuhan was about 14.18 million. According to the statistics, Wuhan received a total of 55.1 million face-masks (including N95 masks and medical-surgical masks) during the period from February 3, 2020, to February 13, 2020. Therefore, we set the daily effective proportion of wearing masks was 0.1 and the intervention time of self-protection measure was the time when a group of pneumonia cases with unknown etiology were first published in Wuhan municipal health commission (January 11, 2020). The results are shown in Fig.9. 
< Figure 9 The change curve of newly reported cases per day in Wuhan under actual interventions >

Fig.9 shows that: the original propagation curve continuously shrunk to the actual epidemic curve of Wuhan and the final result was almost consistent with the actual curve of Wuhan under the three interventions, which also showed the effectiveness of the model in this study. Among them, quarantine measure was the most effective, and medical reception-cured measure and self-protection measure were mainly reflected in reducing the peak of infection and delaying the spread of the COVID-19 in the early stage (the curve moved to the left). However, there is some deviation between the final curve and the actual curve of Wuhan, which is mainly because of the following reasons: 1) it has a certain difference that the original spread trend of the COVID-19 estimated by the early data of other countries brought into Wuhan city itself; 2) there will be some errors in the case detection and data recording in Wuhan city because of the large amount of unknown information about the new virus in the early stage; 3 ) there is still an obvious difference between the distribution of population and the actual situation, such as there is no crowd activity for the lakes, fields, and wasteland.

\section{Discussion}

Facing the increasingly serious threat of COVID-19, all countries urgently need to use computer modeling to determine the best strategy to mitigate the impact of COVID-19 ${ }^{[5]}$. The type of intervention measures, the intensity of implementation, the scope of implementation, and the intervention time are different in different countries because of the differences of the geographical environment factors such as the evolution state of the epidemic, urban building distribution, human's lifestyle, and economic development, etc. However, this is also the key to the effectiveness of COVID-19 prevention and control. We aim to put forward a model that can integrate spatial and temporal information to further consider the development law of COVID-19 under the influence of more complex natural and social factors, to master more deep information about the spatiotemporal spread pattern of the COVID-19 and epidemic prevention and control. Meanwhile, it also can explain the practical significance of the model parameters by mapping the model parameters to the geographical space, which can provide more direct instructive information for the implementation of specific invention policies after scientifically and reasonably evaluating and quantifying the potential of interventions. But this study has several notable limitations:

Due to the existing data on epidemic cases were not complete, we believed that there were still many uncertainties in the research on the mechanism of COVID-19 dynamic spread, such as the proportion of 
asymptomatic infected people and the asymptomatic spread rate. Although the model parameters of this study referred to the research of articles in famous international journals ${ }^{[1,16,54]}$, a large number of unknown parameters were ineluctably included in the quantitative model of COVID-19. However, it was worth noting that our focus was not on the forecast of the epidemic situation in various regions, and the curve parameters of the COVID-19 dynamics model were mainly used to evaluate and quantify the impact of interventions. The relevant conclusions were consistent with recent studies ${ }^{[5,13,16,23]}$. Besides, due to the lack of spatiotemporal attribute data such as population data, traffic data, building data, and human migration data, we had not established the relationship between the grid and corresponding geographic attributes. Therefore, the population distribution and COVID-19 spread model in this study was a kind of approximate simulation of the actual situation combining with the objective law from the perspective of the whole, which aimed to prepare for the subsequent evaluation of the potential of interventions. Meanwhile, the author indicates that even if the detailed epidemic data can be obtained, it only includes the location where patients showed symptoms, but the location of the infection is still not known, in other words, the current spread models of the COVID-19 have the problem of scientific. Nevertheless, our results for the spread of the COVID-19 were consistent with a recent work in which predicted the epidemic situation in different regions based on the SEIRD model ${ }^{[55]}$.

This study preliminarily revealed objectively the spatiotemporal spread distribution law and development trend of COVID-19 from the perspective of geography and established the relationship between the model parameters and the actual geographical significance. We intended to use the available data to simulate the more complex phenomenon of the spread of COVID-19 in reality, such as data of urban travel and crowd migration data, to enhance the authenticity of the model although complete observation data was difficult to obtain. In future work, we hope to solve the problem of scientific verification of interventions model through multi-source data, such as network news data, global event database, and remote sensing data. The model also considered the impact of hospitals' spatial distribution on the prevention and control of the COVID-19, which also provided a possible way to analyze the problem about the medical resource allocation, spatial location selection, and overall planning of medical admission in different regions. Finally, the mapping between social-economic data and grid areas can be established to quantify the cost of economy and resources under different interventions, which provides more guidance for scientific decision-making and accurate implementation for prevention and control of COVID-19. To 
sum up, these will be the focus and difficulty of our subsequent research.

\section{Conclusion}

In summary, this study combined the COVID-19 dynamic spread model with geography from a new perspective to quantitatively analyze the impacts of prevention and control measures. The discrete grid was used to divide the geographical regions and the spatiotemporal spread model of COVID-19 was designed based on the correlation between infected cases, hospital, and the grid. Meanwhile, the model parameters of interventions were mapped to the corresponding grid regions, and finally, the COVID-19 dynamic model was used for quantitative analysis. Through the simulation of the original spread status of COVID-19 and the actual epidemic data in Wuhan, the conclusions are as follows:

1) Quarantine measure was the most effective measure for epidemic prevention and control. The main role of the quarantine measure was to reduce the number of infection peaks, advance the arrival time of the peak, and shorten the duration of the epidemic, especially for infectious diseases with high infectivity. However, the quarantine measure must be effective under sufficient intensity, and the effect of the quarantine measure would become lower with the delay of intervention time. Besides, strict quarantine measure may be avoided in the early stages of the outbreak which the spread of the epidemic is mild.

2) Medical reception-cured measure played mainly a part in the early stage of the COVID-19 outbreak. The increase of medical beds could significantly reduce the peak of the epidemic but had little effect on the arrival time of peak and duration of the epidemic. Besides, the early intervention time could significantly delay the arrival of the infection peak. However, the outbreak would still occur again and the scale was similar to the original infectious state when the medical beds reached full load.

3) Self-protection measures could reduce the number of peak and delay the arrival time of the peak and duration of the epidemic, which could give the government more buffer time. However, the self-protection measure almost had no effects under the strict quarantine measure. Besides, the medical resources could be concentrated in hospitals and other places in urgent need under strict quarantine measure.

4) The model in this study could analyze qualitatively and quantitatively the impacts of quarantine measure, self-protection measure, and medical reception-cured measure to slow the spread of epidemic considering spatial information of the COVID-19, which was scientific and reasonable. Meanwhile, it had high interpretability for the practical significance for intervention model parameters and could map the model to the actual geographical area, which is helpful for the scientific formulation of specific epidemic 
prevention and control decision.

532

533

534

535

536

537

538

539

540

\section{Abbreviations}

COVID-19: Coronavirus disease 2019; SEIQR: susceptible - exposed - infected - quarantined recovered; SIR-X: susceptible-infectious-X; SIQR: Susceptible - Infectious - Quarantined - Recovered; SEIRD: susceptible - exposed - infected - recovered - deceased; SEIRS: susceptible - exposed - infectious recovered - susceptible; e-ISHR: exposed - infected - susceptible - hospital - recovered; ENM: ecological niche models; CT: Computed tomography; CSSE: Center for Systems Science and Engineering.

\section{Acknowledgments}

$$
\text { None. }
$$

\section{Authors' contributions}

Wen Cao and Haoran Dai in Conceptualization, methodology, Writing - original draft, Writing review \& editing. Xiaochong Tong in Funding acquisition. Jingwen Zhu, Yuzhen Tian and Feilin Peng in providing critical feedback and edits to subsequent revisions.

\section{Funding}

This study was funded by the National Key Research and Development Program of China (2018YFB0505304) and the National Natural Science Foundation of China (Grant No. 41671409).

\section{Availability of data and materials}

$$
\text { Not applicable. }
$$

\section{Ethics approval and consent to participate}

Not applicable.

\section{Consent for publication}

Not applicable.

\section{Competing interests}

$$
\text { The authors declare no conflicts. }
$$

\section{Authors details}

${ }^{1}$ School of Geoscience and Technology, Zhengzhou University, Zhengzhou, China

${ }^{2}$ School of Geospatial Information, University of Information Engineering, Zhengzhou, China

These authors contributed equally to this article and share first authorship. 


\section{References}

560 [1] Chen N, Zhou M, Dong X, et al. Epidemiological and clinical characteristics of 99 cases of 2019 novel coronavirus pneumonia in Wuhan, China: a descriptive study. The Lancet. 2020; 395 (10223): 507-513. https://doi.org/10.1016/S0140-6736(20)30211-7.

[2] Li Q, Guan XH, Wang XY, Wu P, et al. Early spread dynamics in Wuhan, China, of novel coronavirus-infected pneumonia. National England Journal Medicine. 2020; 382: 1199-1207. https://doi.org/10.1056/NEJMoa2001316.

[3] Tian HY, Liu YH, Li YD, et al. An investigation of spread control measures during the first 50 days of the COVID-19 epidemic in China. Science. 2020; 6491(368): 638-642. https://doi.org/10.1126/science.abb6105.

[4] Chan J, Yuan SF, Kok K, et al. A family cluster of pneumonia associated with the 2019 novel coronavirus indicating person - to person spread: a study of a family cluster. The lancet. 2020; 395 (10223): 514-523. https://doi.org/10.1016/S0140-6736(20)30154-9.

[5] Currie CSM., Fowler JW, Kotiadis K, et al. How simulation modeling can help reduce the impact of COVID-19. Journal of simulation. 2020; 2(14): 83-97. https://doi.org/10.1080/17477778.2020.1751570.

[6] Wu JT, Leung K., Leung GM. Nowcasting and forecasting the potential domestic and international spread of the 2019-nCoV outbreak originating in Wuhan, China: a modelling study. The Lancet. 2020; 10225(395): 689-697. https://doi.org/10.1016/S0140-6736(20)30260-9.

[7] Wang HW, Wang ZZ, Dong YQ, et al. Phase-adjusted estimation of the number of Coronavirus Diseases 2019 cases in Wuhan, China. Cell Discovery. 2020; 6: 10. https://doi.org/10.1038/s41421-020-0148-0.

[8] Zhao S, Lin QY, Ran JJ, et al. Preliminary estimation of the basic reproduction number of novel coronavirus (2019-nCoV) in China, fro-m 2019 to 2020: A data-driven analysis in the early phase of the outbreak. International Journal of Infectious Diseases. 2020; 92: 214-217. https://doi.org/10.1016/j.ijid.2020.01.050.

[9] Zhou T, Liu Q, Yang Z, et al. Preliminary prediction of the basic reproduction number of the Wuhan novel coronavirus 2019-nCoV. Journal Evidence-Based Medicine. 2020; 13: 3-7. https://doi.org/10.1111/jebm. 12376.

[10] Read J.M, Bridgen JRE, Cummings DAT, et al. Novel coronavirus 2019-nCoV: early estimation of epidemiological parameters and epidemic predictions. medRix 2020.01.23.20018549. https://doi.org/10.1101/2020.01.23.20018549.

[11] Jumpen W, Wiwatanapataphee B, Wu YH, et al. A SEIQR model for pandemic influenza and its parameter identification. International Journal of Pure and Applied Mathematics. 2020; 52(2): 247-265. http://ijpam.eu/contents/2009-52-2/8/index.html.

[12] Maier BF, Brockmann D. Effective containment explains sub-exponential growth in confirmed cases of recent COVID-19 outbreak in Mainland China. Science. 2020; 368(6492): 742-746. https://doi.org/10.1126/science.abb4557.

[13] Crokidakis N. COVID-19 spreading in Rio de Janeiro, Brazil: Do the policies of social isolate-on really work?. Chaos, Solitons \& Fractals. 2020; 136: 109930. https://doi.org/10.1016/j.chaos.2020.109930.

[14] Viguerie A, Lorenzo G, Auricchio F, et al. Simulating the spread of COVID-19 via a spatially-resolved susceptible - exposed infected - recovered - deceased (SEIRD) model with heterogeneous diffusion. Applied Mathematics Letters. 2020; 111: 106617. https://doi.org/10.1016/j.aml.2020.106617.

[15] Cooke KL, Driessche PVD. Analysis of an SEIRS epidemic model with two delays. Journal of Mathematical Biology. 1996; 35(2): 240-260. https://doi.org/10.1007/s002850050051. 
[16] Okuonghae D, Omame A. Analysis of a mathematical model COVID-19 population dynamics in Lagos, Nigeria. Chaos, Solitons \& Fractals. 2020; 139: 110032. https://doi.org/10.1016/j.chaos.2020.110032.

[17] Davies NG, Klepac P, Liu Y, et al. Age-dependent effects in the spread and control of COVID-19 epidemics. Nature Medicine. 2020; 26: 1205-1211. https://doi.org/10.1038/s41591-020-0962-9.

[18] Davies NG, Kucharski AJ, Eggo RM, et al. Effects of non-pharmaceutical interventions on COVID-19 cases, deaths, and demand for hospital services in the UK: a modelling study. The Lancet. 2020; 7(5): e375-e385. https://doi.org/10.1016/S2468-2667(20)30133-X.

[19] Kassa SM, Njagarah JBH., Terefe YA. Analysis of the mitigation strategies for COVID-19: From mathematical modelling perspective. Chaos, Solitons \& Fractals. 2020; 138: 109968. https://doi.org/10.1016/j.chaos.2020.109968.

[20] Stadnytskyi V, Bax CE, Bax A, et al. The airborne lifetime of small speech droplets and their potential importance in SARS-CoV-2 spread. PNAS. 2020; 117(22): 11875-11877. https://doi.org/10.1073/pnas.2006874117.

[21] Ribeiro MHDM, Silva RGD, Mariani VC, et al. Short-term forecasting COVID-19 cumulative confirmed cases: Perspectives for Brazil.Chaos, Solitons \& Fractals. 2020; 135:109853. https://doi.org/10.1016/j.chaos.2020.109853.

[22] Chakraborty T, Ghosh I. Real-time forecasts and risk assessment of novel coronavirus (COVID-19) cases: A data-driven analysis. Chaos, Solitons \& Fractals, 2020, 135: 109850. https://doi.org/10.1016/j.chaos.2020.109850.

[23] Li SJ, Song K, Yang BR, et al. Preliminary assessment of the COVID-19 outbreak using 3-staged model e-ISHR. Journal Shanghai Jiaotong University (Science). 2020; 25(2): 157-164. https://doi.org/10.1007/s12204-020-2169-0.

[24] Zhang LY, Li DC, Ren JL. Analysis of COVID-19 by Discrete Multi-stage Dynamics System with Time Delay. Geomatics and Information Science of Wuhan University. 2020; 45(5): 658-666. https://doi.org/10.13203/j.whugis20200206.

[25] Lymperopoulos IN. \#stayhome to contain contain COVID-19: Neuro-SIR-Neuro dynamical epidemic modeling of infection patterns in social networks. Expert Systems with Applications 165,113970. https://doi.org/10.1016/j.eswa.2020.113970.

[26] Bouchnita, A., Jebrane, A., 2020. A hybrid multi-scale model of COVID-19 spread dynamics to assess the potential of non-pharmaceutical interventions. Chaos, Solitons \& Fractals. 2020; 138: 109941. https://doi.org/10.1016/j.chaos.2020.109941.

[27] Lin QY, Zhao S, Gao DZ, et al. A conceptual model for the coronavirus disease 2019 (COVID-19) outbreak in Wuhan, China with individual reaction and governmental action. International Journal of Infectious Diseases. 2020; 93: 211-216. https://doi.org/10.1016/j.ijid.2020.02.058.

[28] Fang YQ, Nie YT, Penny M. Spread dynamics of the COVID-19 outbreak and effectiveness of government interventions: A data-driven analysis. Journal of Medical Virology. 2020; 92(6): 645-659. https://doi.org/10.1002/jmv.25750.

[29] Tang B, Wang X, Li Q, et al. Estimation of the spread risk of the 2019-nCoV and its impaction for public health interventions. Journal of Clinical Medicine. 2020; 9(2): 462. https://doi.or g/10.3390/jcm9020462.

[30] Chu DK, Duda S, Solo K, et al. Physical distancing, Face masks, and eye protection to prevent person-to-person spread of SARS-CoV-2 and COVID-19: A systematic review and meta-analysis. Lancet. 2020; 72(4): 1500. https://doi.org/10.1016/j.jvs.2020.07.040.

[31] Ma ZH, Wang SF, Li XH. A generalized infectious model induced by the contacting distance (CTD). Nonlinear Analysis: Real World Applications. 2020; 54: 103113. https://doi.org/10.1016/j.nonrwa.2020.103113. 
[32] Gostic K, Gomez ACR, Mummah RO, et al. Estimated effectiveness of symptom and risk screening to prevent the spread of COVID-19. eLife 2020; 9: e55570. https://doi.org/10.7554/eLife.55570.

[33] Wells C.R. Sah, P, Moghadas SM, et al. Impact of international travel and border control measures on the global spread of the novel 2019 coronavirus outbreak. PANS. 2020; 117(13): 7504-7509. https://doi.org/10.1073/pnas.2002616117.

[34] Hellewell JH, Abbott S, Gimma A, et al. Feasibility of controlling COVID-19 outbreaks by isolation of cases and contacts. Global Health. 2020; 4(8): e488-e496. https://doi.org/10.1016/S2214-109X(20)30074-7.

[35] Wölfel R, Corman VM, Gugemos W, et al.Virological assessment of hospitalized patients with COVID-2019. Nature. 2020; 281: 465-469. https://doi.org/10.1038/s41586-020-2196-x.

[36] Rosa GL, Bonadonna L, Lucentini Luca, et al. Coronavirus in water environments: Occurrence, persistence and concentration methods - A scoping review. Water Research, 2020; 179: 115899. https://doi.org/10.1016/j.watres.2020.115899.

[37] Leclerc QJ, Fuller NM, Knight LE. et al. What settings have been linked to SARS-CoV-2spread cluster? [version 1; peer review:1 approved with reservations]. Wellcome Open Research, 2020; 5: 83. https://doi.org/10.12688/wellcomeopenres.15889.1.

[38] Lu JY, Gu, Li JN, Xu KB, CH, et al. COVID-19 outbreak associated with Air conditioning in restaurant, Guangzhou, China, 2020. Emerging Infectious Diseases. 2020; 26(7): 1682-1631. https://dx.doi.org/10.3201/eid2607.200764.

[39] Morawska L, Cao JJ. Airborne spread of SARS-CoV-2: The world should face the reality. Environment International. 2020; 139: 105730. https://doi.org/10.1016/j.envint.2020.105730.

[40] Dehning J, Zierenberg J, Spitzner FP, et al. Inferring change point in the spread of COVID-19 reveals the effectiveness of interventions. Science. 2020; 6500(369): eabb9789. https://doi.org/10.1126/science.abb9789.

[41] Thu TPB, Ngoc PNH., Hai NM, et al. Effect of the social distancing measures on the spread of COVID-19 in 10 highly infected countries. Science of the Total Environment. 2020; 742: 140430. https://doi.org/10.1016/j.scitotenv.2020.140430.

[42] Barmparis GD, Tsironis GP. Estimating the infection horizon of COVID-19 in eight counties with a data-driven approach. Chaos, Solitons \& Fractals. 2020; 135: 109842. https://doi.org/10.1016/j.chaos.2020.109842.

[43] Han WG. Data-driven and model-driven spatiotemporal data ming-respective case study in traffic flow data and epidemic data, Chinese Academy of Sciences. 2006. http://ir.igsnrr.ac.cn/handle/311030/106

[44] Franch-Pardo I, Napoletano BM, Rosete-Verges F, et al. Spatial analysis and GIS in the study of COVID-19. A review. Science of the Total Environment. 2020; 739: 140033. https://doi.org/10.1016/j.scitotenv.2020.140033.

[45] Feng MX, Fang ZX, Lu XB, et al. Traffic Analysis Zone-Based Epidemic Estimation Approach of COVID-19 Based on Mobile Phone Data: An Example of Wuhan. Geomatics and Information Science of Wuhan University. 2020, 45(5): 651-657, 681. https://doi.org/10.13203/j.whugis20200141.

[46] Sugg MM, Spaulding RJ, Lane SJ, et al. Mapping community-level determinants of COVID-19 spread in nursing homes: A multi-scale approach. Science of the Total Environment. 2020, 752: 141946. https://doi.org/10.1016/j.scitotenv.2020.141946.

[47] Ren HY, Zhao L, Zhang A, et al. Early forecasting of the potential risk zones of COVID-19 in China's megacities. Science of the Total Environment. 2020; 729: 138995. https://doi.org/10.1016/j.scitotenv.2020.138995.

660 [48] Fang ZX. Thinking and challenges of crow dynamics observation from the perspectives of public health and public security. 
[49] Cao ZD. Mathematical modeling and spatial analysis of spatiotemporal data-case study based on SARS epidemic in Guangzhou. Chinese Academy of Sciences. 2008. http://ir.igsnrr.ac.cn/handle/311030/1774.

664 [50] Dong E, Du H, Gardner L. An interactive web-based dashboard to track COVID-19 in real time. Lancet Infectious Diseases. 2020; 20(5): 422-534. https://doi.org/10.1016/S1473-3099(20)30120-1.

[51] Cui HJ, Hu T. Nonlinear regression in COVID-19 forecasting (in Chinese). SCIENTIA SINICA Mathematica. 2020; (50): 1-12. https://engine.scichina.com/doi/10.1360/SSM-2020-0055.

[52] Xia JZ, Zhou Y, Li Z, et al. COVID-19 risk assessment driven by urban spatiotemporal big data: case study of Guangdong-Hong Kong-Macao Greater Bay Area. Acta Geodaetica et Cartographica Sinica. 2020; 49(6): 671-680. http://xb.sinomaps.com/CN/10.11947/j.AGCS.2020.20200080.

[53] Alberti T, Faranda D. On the uncertainty of real-time predictions of epidemic growths: A COVID-19 case study for China and Italy. Communications in Nonlinear Science and Numerical Simulation. 2020; 90: 105372. https://doi.org/10.1016/j.cnsns.2020.105372.

[54] Guo L, Yang WQ, Bi YF. Research on the Scale of Daily Life Unit Based on Residents' Travel Characteristics. China Academic Journal Electronic Publishing House. 2016; 1004-1015. https://kns.cnki.net/KCMS/detail/detail.aspx?dbcode=CPFD\&filename=CSJT201604001098.

[55] Cauchemez S, Fraser C, Wan MD, et al. Middle east respiratory syndrome coronavirus: quantification of the extent of the epidemic, surveillance biases, and transmissibility. The Lancet Infectious Diseases. 2020; 1(14): 50-56. https://doi.org/10.1016/S1473-3099(13)70304-9.

[56] Viguerie A, Lorenzo G, Auricchio F, et al. Simulating the spread of COVID-19 via a spatially-resolved susceptible - 
Figures
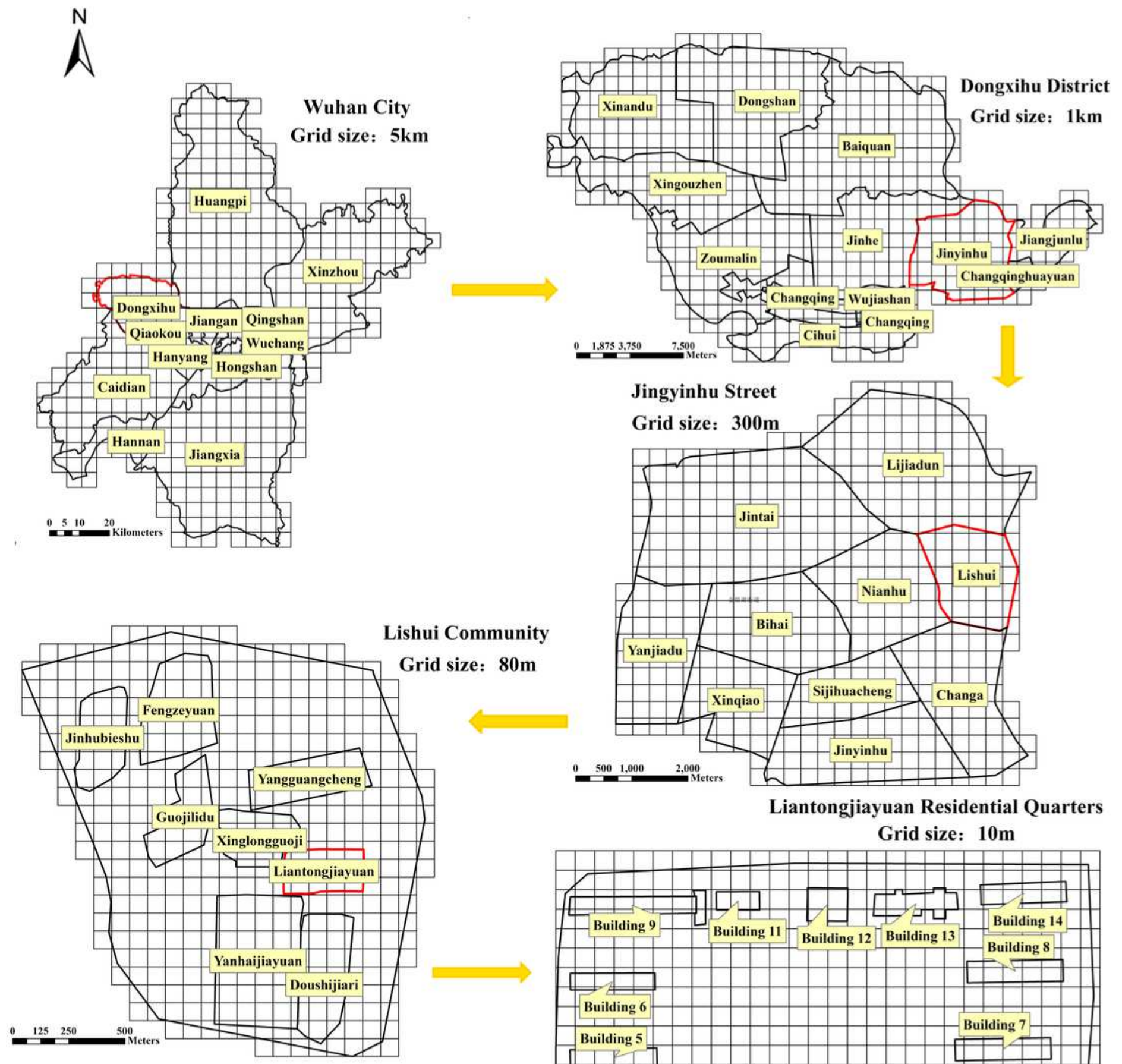

Liantongjiayuan Residential Quarters Grid size: $10 \mathrm{~m}$

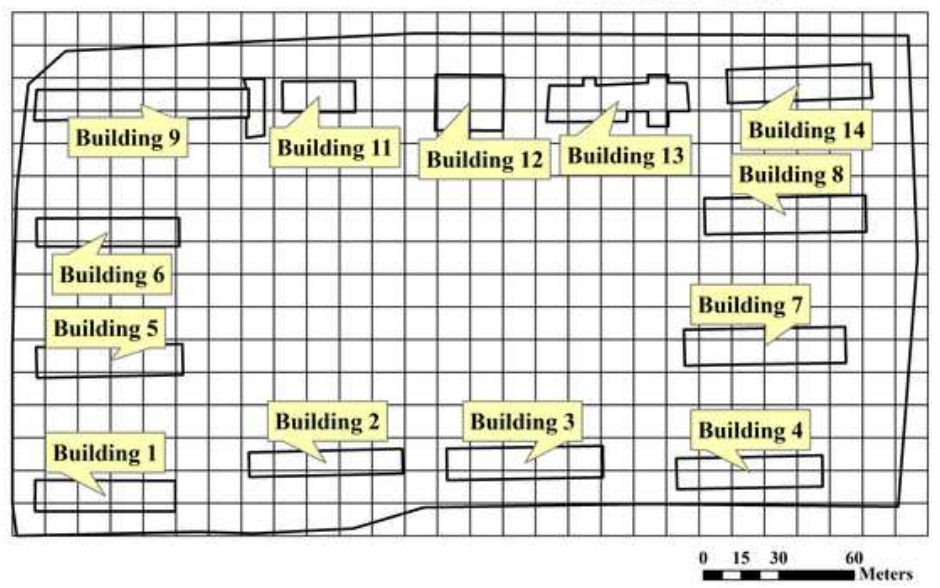

\section{Figure 1}

Grid filling diagram of traditional prevention and control unit. Note: The designations employed and the presentation of the material on this map do not imply the expression of any opinion whatsoever on the part of Research Square concerning the legal status of any country, territory, city or area or of its 
authorities, or concerning the delimitation of its frontiers or boundaries. This map has been provided by the authors.
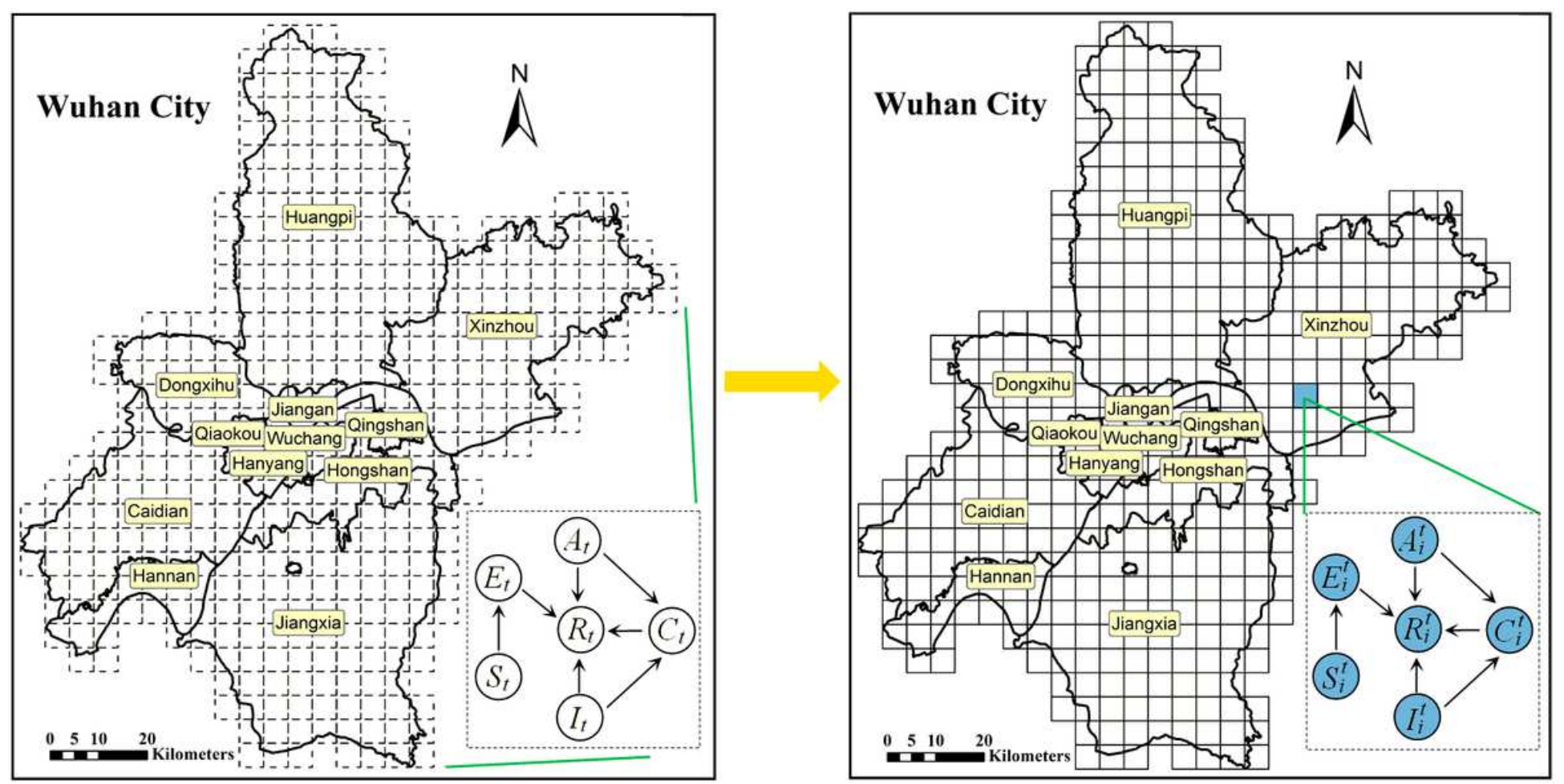

\section{Figure 2}

Schematic diagram of the quarantine measure model based on a discrete grid. Note: The designations employed and the presentation of the material on this map do not imply the expression of any opinion whatsoever on the part of Research Square concerning the legal status of any country, territory, city or area or of its authorities, or concerning the delimitation of its frontiers or boundaries. This map has been provided by the authors. 


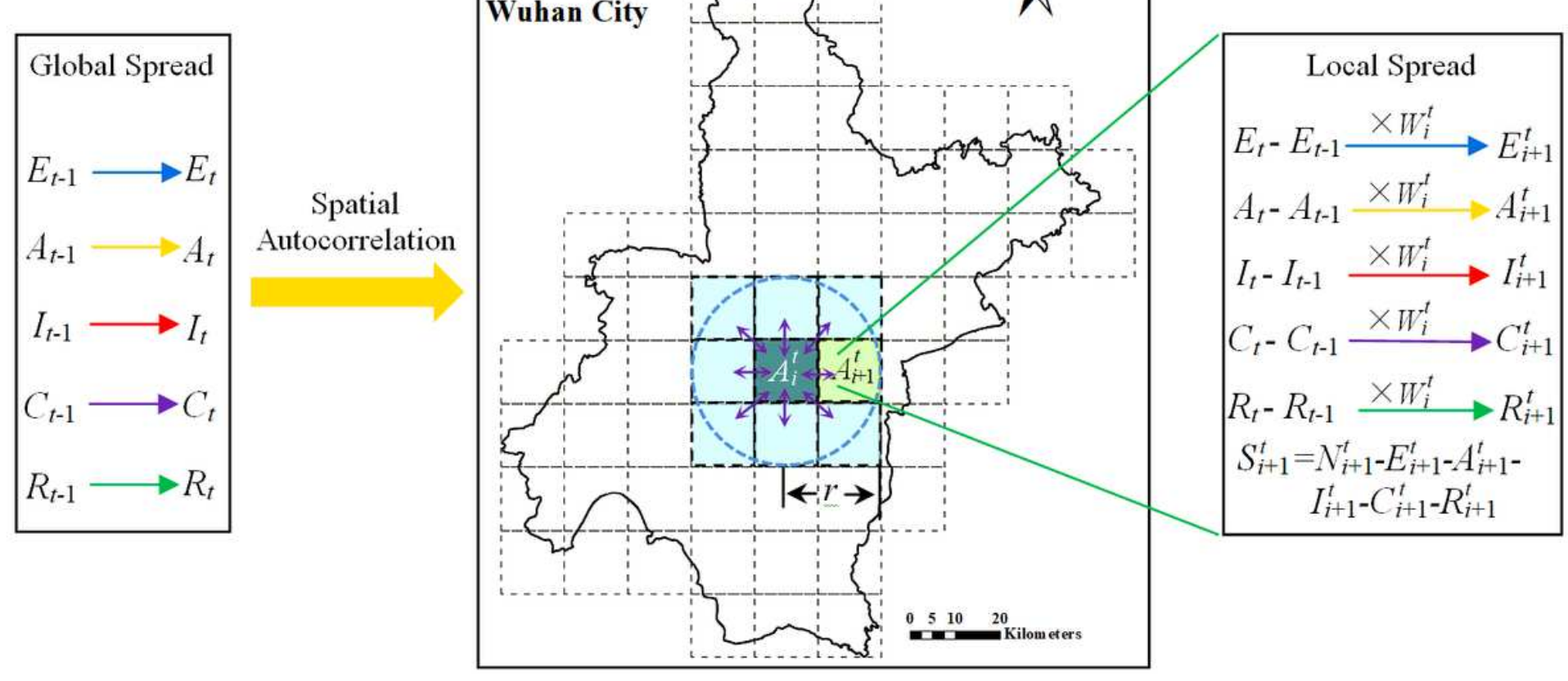

Figure 3

Spatiotemporal spread model for infectious diseases based on a discrete grid. Note: The designations employed and the presentation of the material on this map do not imply the expression of any opinion whatsoever on the part of Research Square concerning the legal status of any country, territory, city or area or of its authorities, or concerning the delimitation of its frontiers or boundaries. This map has been provided by the authors. 


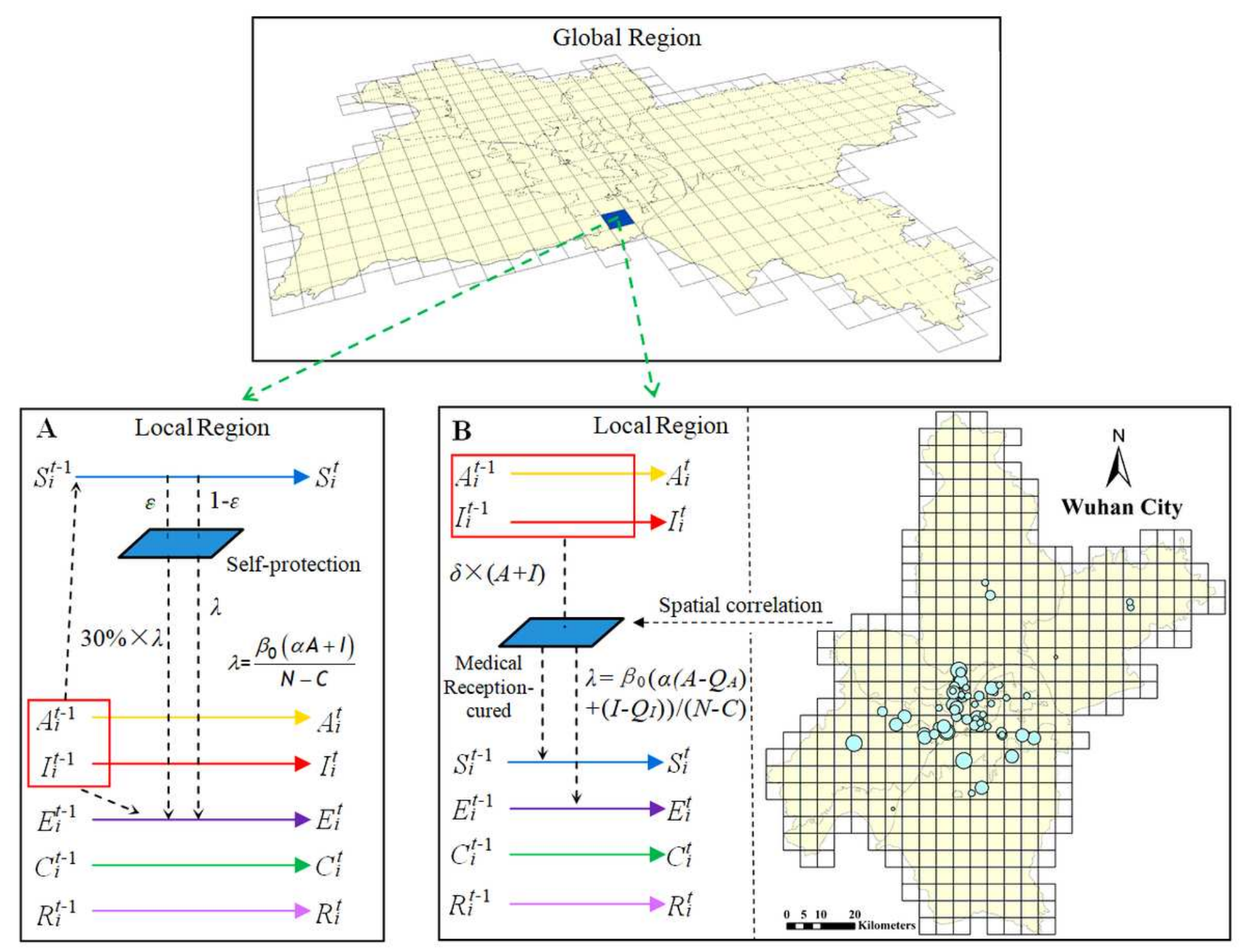

Figure 4

Schematic diagram of medical reception-cured measure (A) and self-protection measure (B). Note: The designations employed and the presentation of the material on this map do not imply the expression of any opinion whatsoever on the part of Research Square concerning the legal status of any country, territory, city or area or of its authorities, or concerning the delimitation of its frontiers or boundaries. This map has been provided by the authors. 


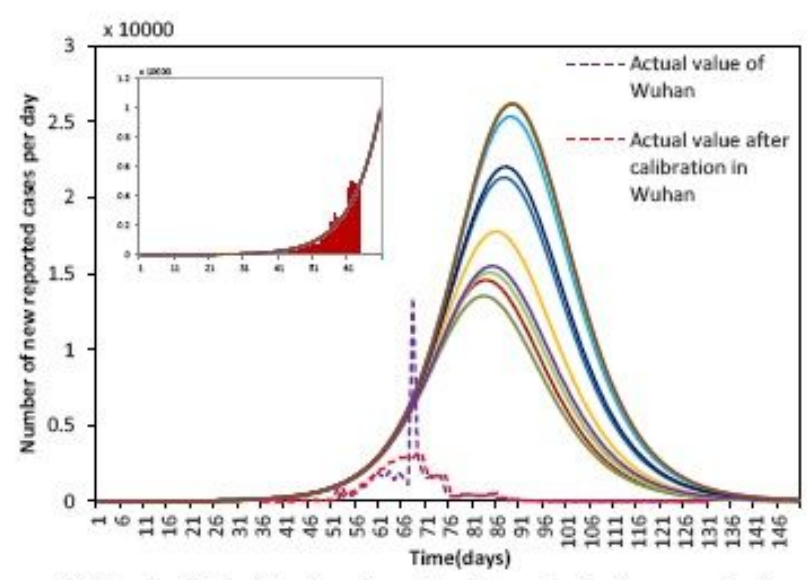

(a)Early UK data to simulate the original spread of COVID-19

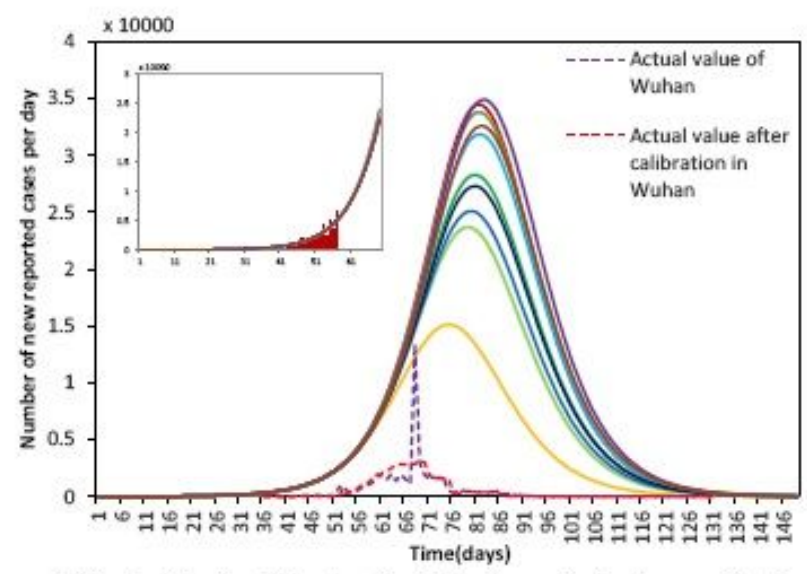

(c)Early Spain data to simulate the original spread of COVID-19

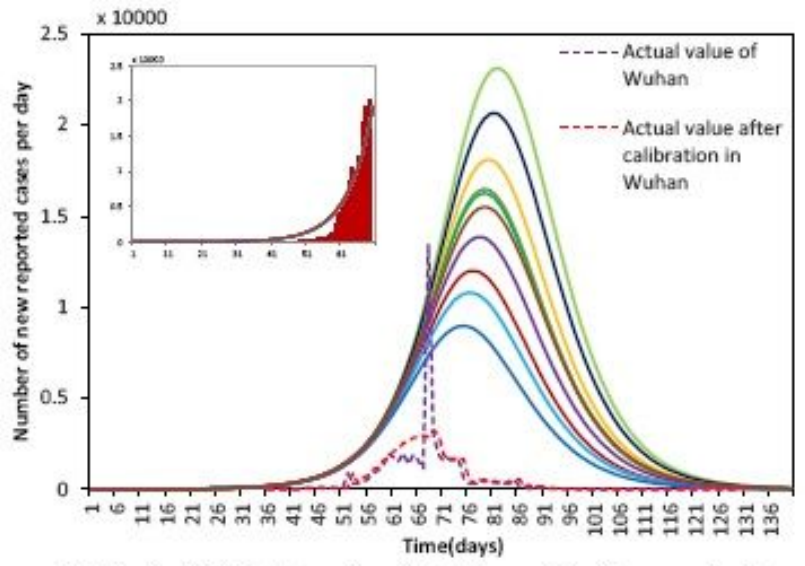

(b)Early US data to simulate the original spread of COVID-19

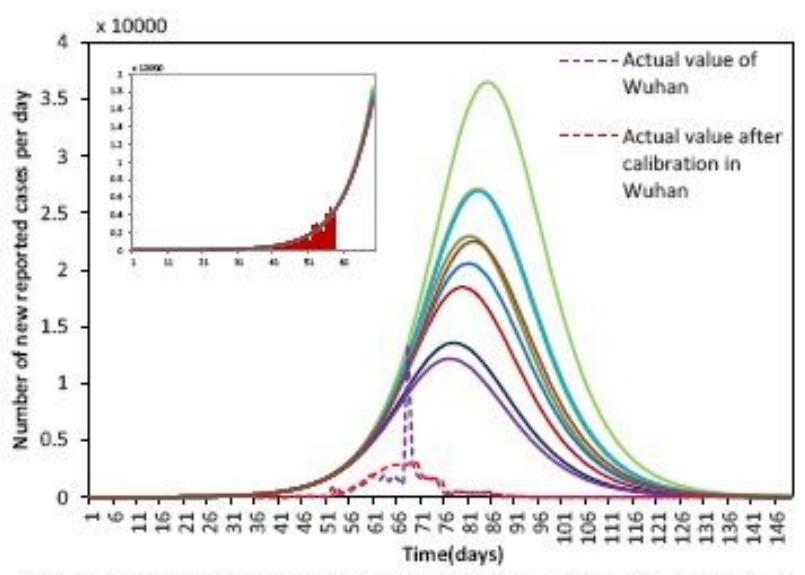

(d)Early German data to simulate the original spread of COVID-19

\section{Figure 5}

The number of newly reported cases per day of original state simulated by genetic algorithm (Small chart section: the red histogram represents the early epidemic data of each country, and the curve is the original spread state curve of COVID-19 in each country. Here, the starting time of X coordinate in the UK, the US, Spain, and Germany is January 31, 2020, January 20, 2020, February 1, 2020, and January 27, 2020, respectively; big graph section: the solid-line curve is the original spread state curve of Wuhan. The red dotted-line curve shows the actual number of newly reported cases in Wuhan after calibration, and the purple dotted-line curve represents the actual number of newly reported cases in Wuhan. Here, the starting time of $\mathrm{X}$ coordinate in the Wuhan is December 28, 2020 [9] 

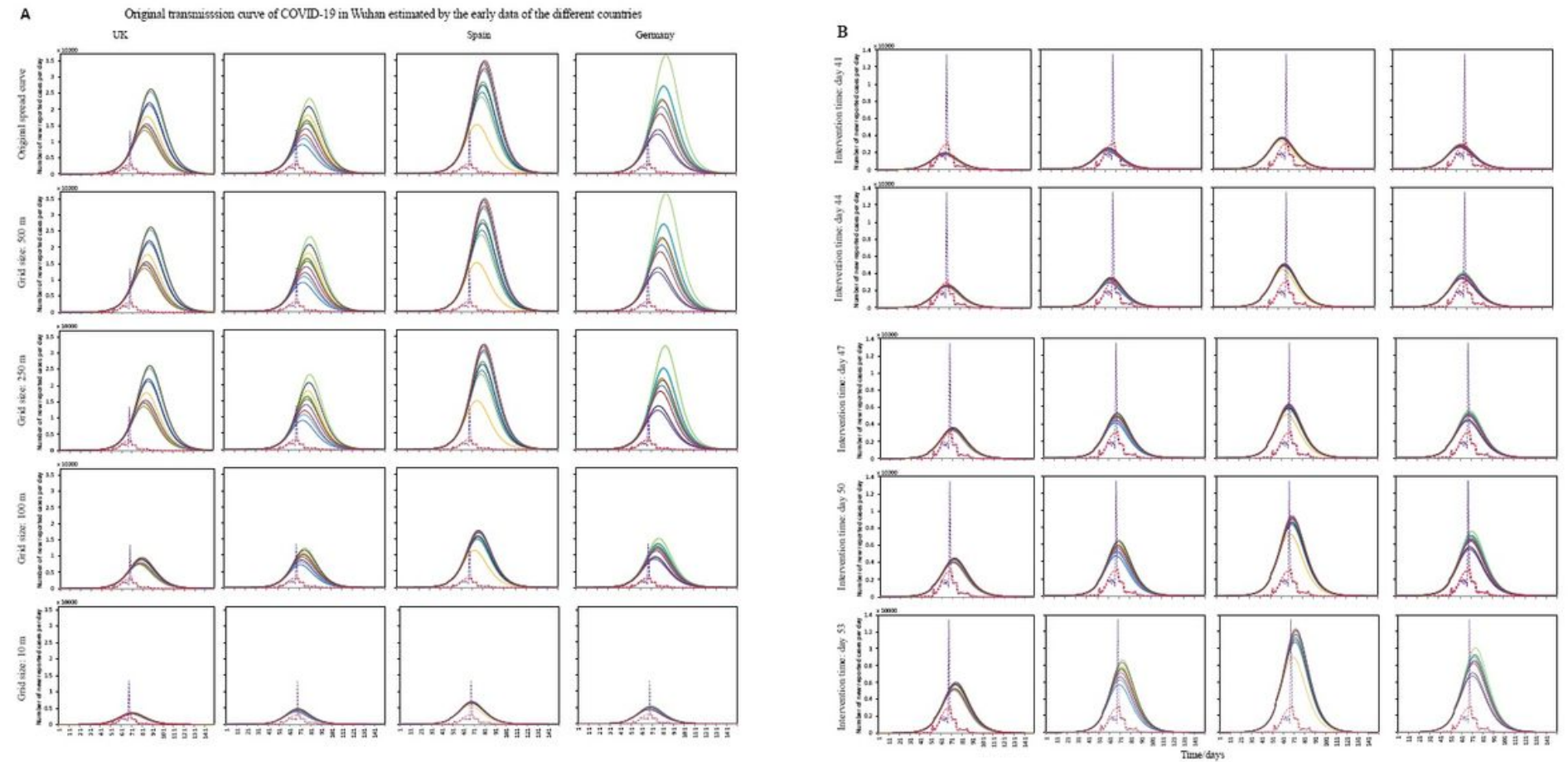

Figure 6

The change curve of newly reported cases per day in Wuhan under different quarantine measure
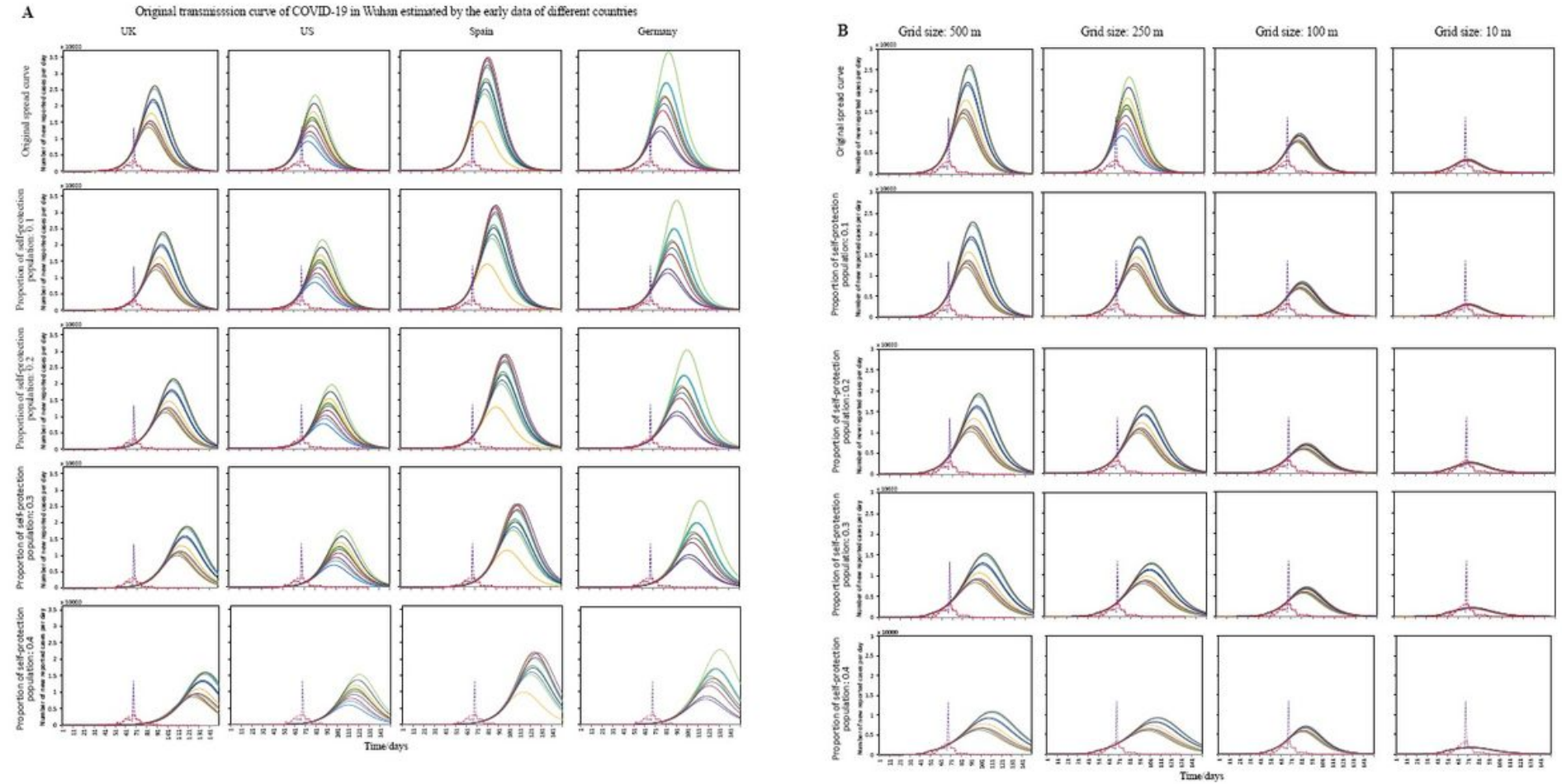

\section{Figure 7}

The change curve of newly reported cases per day in Wuhan under different self-protection measure 

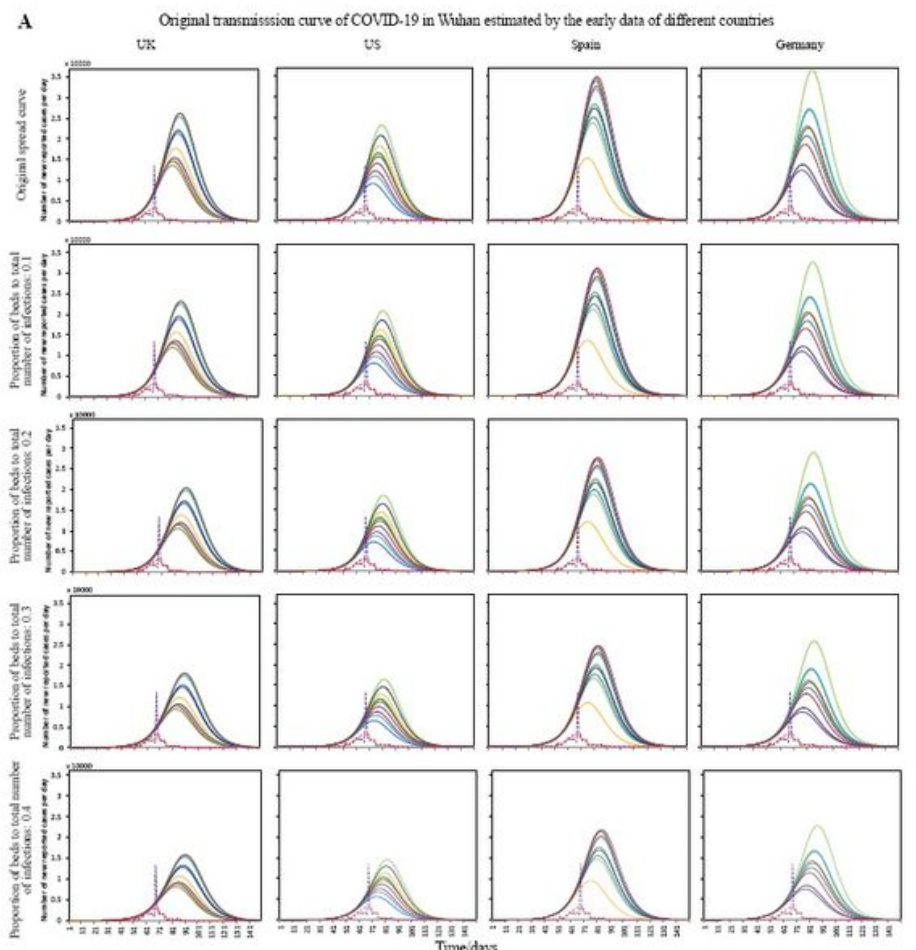

B
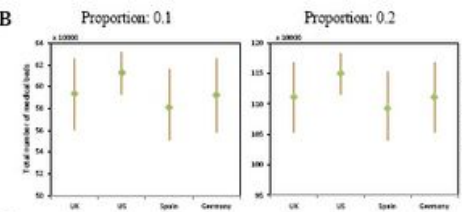

Proportical: 0.3
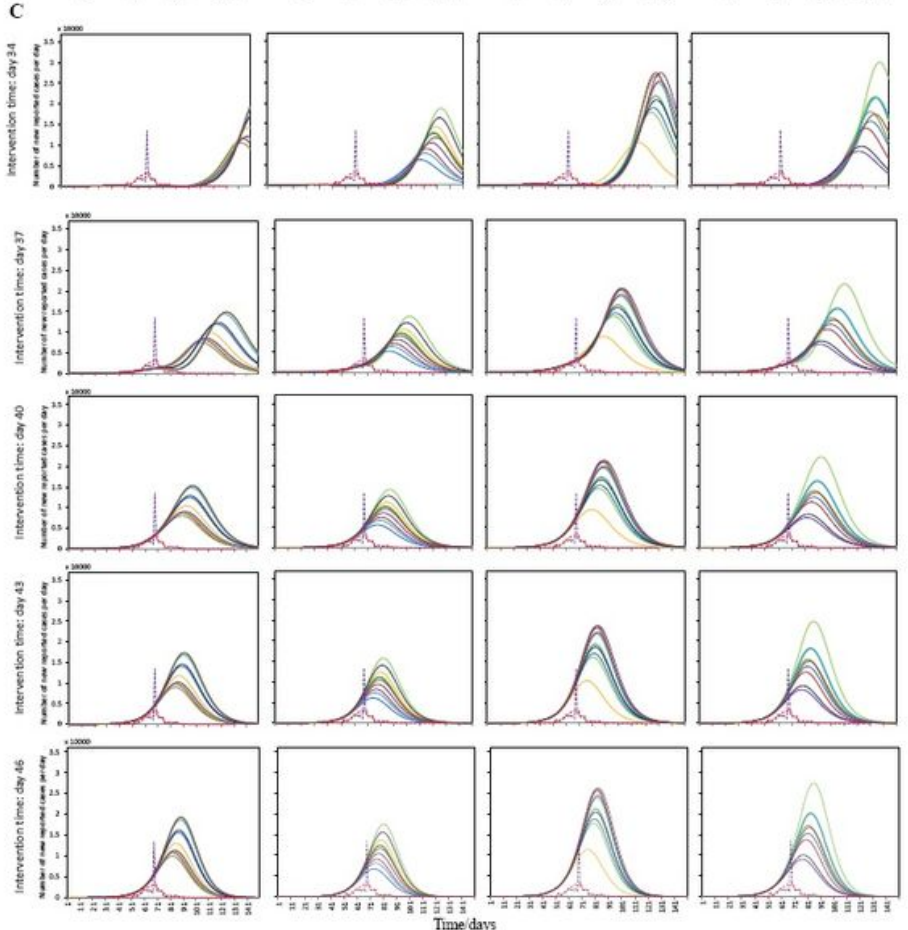

Figure 8

The change curve of newly reported cases per day in Wuhan under the different medical reception-cured measure (the length of the yellow vertical line and the green prism points indicates the $95 \%$ confidence interval and the median values of the total number of invested beds corresponding to 10 curves in group $B$, respectively) 

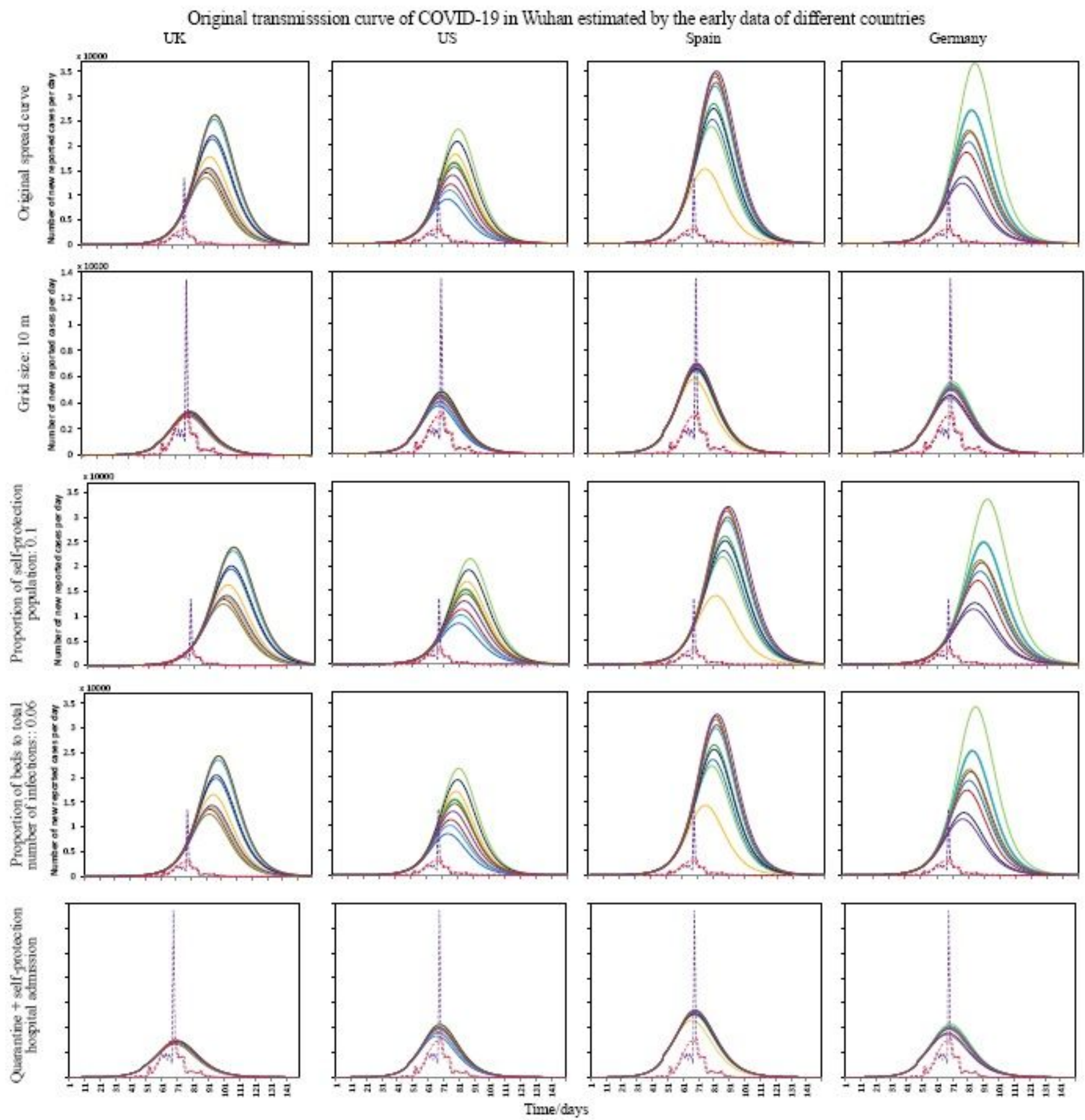

Figure 9

The change curve of newly reported cases per day in Wuhan under actual interventions

\section{Supplementary Files}

This is a list of supplementary files associated with this preprint. Click to download. 
- Table.doc 